\title{
Optimization of corrosion protection economics
}

\author{
V. I. Vigdorovich, L. E. Tsygankova and N. V. Shell \\ Derzhavin State University, Internatsyonalnaya Str., 33, Tambov, 392000, \\ Russian Federation.E-mail: vits21@,mail.ru
}

\begin{abstract}
An equation relating corrosion losses and expenditures for anti-corrosion measures is suggested. A formula for the absolute value of the economic efficiency of anti-corrosion protection is introduced.
\end{abstract}

Key words: optimization, protection, corrosion, expenditures, inhibitors, inflation, deflation, function, loss, economics.

Received February 24, 2013.

doi: $\underline{10.17675 / 2305-6894-2013-2-3-245-254}$

\section{Introduction}

As a rule, the methods used for estimation of economic efficiency $(E)$ of expenditures for anti-corrosion measures $(E A C)$ demand a comparison between the base and new variants in terms of practical application and technology [1-3]. A number of factors must be taken into consideration: productivity, safety, efficiency (for example, of corrosion inhibitors or the preservation materials), functional characteristics of structural materials and the raw material resources, the quality of the product being manufactured, and the time factor (in particular, the guaranteed protection period). Undoubtedly, social factors, including the estimation of changes in the ecological situation in the region, are very important. A typical example of this approach is given in [1]. However, the concept using the base variant (BV) includes inevitably subjective elements because this allows one to obtain a result planned in advance (desirable) that is determined by the BV efficiency. An intentionally underestimated BV would give a large economic effect, whereas a better variant would decrease the latter.

A different approach is suggested in [4] in the form of an equation to calculate economic efficiency $E$ of anticorrosion protection

$$
E=\left(K_{\mathrm{dcl}}+K_{\mathrm{icl}}\right)(1-1 / k)-E A C,
$$

where $K_{\mathrm{dcl}}$ and $K_{\mathrm{icl}}$ are the direct and indirect corrosion losses, respectively; $k$ is the efficiency coefficient of anticorrosion protection depending (in our interpretation) on the conditions, the nature of the object being protected, the efficiency of the protective technologies used, the professional skills of the anticorrosion service personnel; $E A C$ are the expenditures for the anticorrosion steps. According to (1), if $k=1$, then $E=-E A C$, i.e., $E A C$ were wasted. If $k \rightarrow \infty, E=\left(K_{\mathrm{dcl}}+K_{\text {icl }}\right)-E A C$. 
The authors of [4] disregard the functional dependence between $E A C$ and $k$ [5]. It should be taken into account that the dependence between $E A C$ and $k$ is not linear because it would contradict the decreasing returns law.

We suggest a method for estimation of the anticorrosion protection efficiency [6-8] free of the above indicated drawbacks. Furthermore, it can in principle be used in many other spheres of human activities. Let us consider it for anticorrosion protection as an example.

\section{Theory of the economic efficiency of anticorrosion measures}

Let us express the relationship between corrosion losses and anticorrosion expenditures by the equation:

$$
K_{\mathrm{tcl}}=K_{\mathrm{scl}}+E A C,
$$

where $K_{\text {tcl }}$ and $K_{\text {scl }}$ are true corrosion losses and strictly corrosion losses, respectively. $E A C$ are considered as part of the $K_{\mathrm{tcl}}$. It reflects the genetic relation between $E A C$ and $K_{\mathrm{scl}}$ and emphasizes that, once used, they are lost as material resources for other spheres of application. Under conditions of efficient anticorrosion protection, an increase in $E A C$ causes an essentially greater decrease in $K_{\mathrm{scl}}$, i.e.

$$
\Delta K_{\mathrm{scl}}>\triangle E A C \text {. }
$$

Taking into account that $K_{\mathrm{scl}}=K_{\mathrm{dcl}}+K_{\text {icl }}$, Eq. (2) tak es the form

$$
K_{\mathrm{tcl}}=K_{\mathrm{dcl}}+K_{\mathrm{icl}}+E A C
$$

$K_{\mathrm{dcl}}$ includes the costs of materials and labour, replacement of corroded structures and mechanisms or their parts, as well as the costs of re-protection (re-preservation). $K_{\text {icl }}$ is understood as losses due to equipment downtime, losses in product, a decrease in product quality, and reduction in the power of machines and mechanisms. Sometimes $K_{\text {icl }}$ cannot be expressed in money terms (when it is a result of explosions or catastrophes resulting in human fatalities).

In the simplest case:

$$
K=\left(K_{\mathrm{dcl}}+K_{\mathrm{icl}}\right) / K_{\mathrm{dcl}}
$$

Further let us use the relationship

$$
K_{\mathrm{dcl}}=f(E A C),
$$

because $K_{\mathrm{dcl}}$ is estimated more easily than $K_{\text {icl }}$ and $K_{\text {scl }}$. Taking Eq. (4) and Eq. (5) into account, one can write:

$$
K_{\mathrm{scl}}=K f(E A C)
$$

The existence of this relationship is doubtless. It is evident that an increase in the efficiency of anticorrosion protection decreases $K_{\mathrm{dcl}}$. The simultaneous increase in the protection efficiency causes an increase in $E A C$ to a certain limit. However, beginning from some $E A C$ value, its further increase will negatively affect the $E$ value. 
The form of the $f(E A C)$ function along with Eq. (2) allow one to estimate economic efficiency $E$ and the decrease in $K_{\mathrm{dcl}}$ and $K_{\mathrm{icl}}$ as a function of $E A C$. Let us consider the rather common case where the same consumer suffers losses $K_{\text {scl }}$ and decreases them at the expense of $E A C$, while the time interval between these values is small. Then $E$ amounts to

$$
E=K_{\text {scl }}^{0}-[K f(E A C)+E A C]
$$

Here and below, the "zero" superscript indicates the values where $E A C=0$.

The real relationship between $E$ and $E A C$ can be described by various functions leading to qualitatively identical results if adapting coefficients are used. These functions should be continuous and single-valued and should satisfy the following conditions:

1) if $E A C=0$, then $E=0$ and $K_{\mathrm{scl}}^{0} / K_{\mathrm{scl}}=1$;

2) $E<K_{\text {scl }}^{0}$ (mathematical formalism leads to $E=K_{\text {scl }}^{0}$ in the limit, which is theoretically unreachable).

3) A single maximum should exist.

The latter condition is obvious: the use of an efficient method of anticorrosion protection leads to a $E$ increase with increasing $E A C$, but $E$ cannot be increased infinitely (the decreasing returns law). Besides, increasing $E A C$ does not lead to a constant $E$ in the limit. Such requirements are satisfied by the function:

$$
K_{\mathrm{scl}}=K \exp \left(-k E A C / K_{\mathrm{dcl}}^{0}\right)
$$

The $E A C / K_{\mathrm{dcl}}^{0}$ ratio reflects the anticorrosion expenditures expressed in $K_{\mathrm{dcl}}^{0}$ units.

The power function can be used:

$$
K_{\mathrm{scl}}=K\left(K_{\mathrm{dcl}}^{0}-E A C\right) k
$$

At $K_{\mathrm{dcl}}^{0}=1$, Eqs. (7) and (8) get simplified:

$$
\begin{gathered}
K_{\mathrm{scl}}=K \exp (-k E A C) \\
K_{\mathrm{scl}}=K(1-E A C) k
\end{gathered}
$$

Let us analyse the relationship between $E$ and $E A C$ taking Eq. (7a) into account. A combination of (7a) and (6) gives:

$$
E=K[1-\exp (-k E A C)-E A C / K]
$$

From Eq. 9:

$$
\begin{aligned}
& \mathrm{d} E / \mathrm{d}(E A C)=\mathrm{Kk} \exp (-k E A C)-1 \\
& \mathrm{~d}^{2} E / \mathrm{d}(E A C)^{2}=-K k^{2} \exp (-k E A C)
\end{aligned}
$$

$\mathrm{d}^{2} E / \mathrm{d}(E A C)^{2}<0$ because $K>0$ and the optimum anticorrosion expenditures $E A C^{*}$ are:

$$
E A C^{*}=(1 / k) \ln (K k)
$$


However, it is only true provided that $k>0$. If $k<0$, then $\mathrm{d} E / \mathrm{d}(E A C)<0$ in accordance with Eq. (10) for any $E A C$ and therefore function (9) has no extremum at these $k$ values. It should also be noted that the case $k<0$ has no practical interest because it corresponds to $K_{\text {scl }}>K_{\text {scl }}^{0}$, hence $E<0$.

Using the sequential approximation method, one can obtain the value $Q$ characterizing the range of anti-corrosive expenditures, where $E>0: 0<E A C<Q$.

The first approximation gives:

$$
Q^{\mathrm{I}}=K[1-\exp (-K k)]
$$

The second approximation results in the expression:

$$
Q^{\mathrm{II}}=K\{1-\exp (-K k[1-\exp (-K k)])\} .
$$

According to [9], $K=2.2-2.5$, i.e. $K_{\mathrm{icl}} / K_{\mathrm{dcl}}=1.2-1.5$. The average $K$ value within Russia is close to 4 [10]. Table 1 shows the $Q^{\mathrm{i}}$ values calculated from the $i$-th approximation at $K=2.5$ and $1[10]$.

Table 1. Relationship between $Q$ and $k$.

\begin{tabular}{cccccccccc}
\hline \multirow{2}{*}{$\boldsymbol{K}$} & $\begin{array}{c}\boldsymbol{i} \text {-th } \\
\text { approximation }\end{array}$ & $\mathbf{0 . 3}$ & $\mathbf{0 . 5}$ & $\mathbf{0 . 8}$ & $\mathbf{1 . 0}$ & $\mathbf{2 . 0}$ & $\mathbf{4 . 0}$ & $\mathbf{6 . 0}$ & $\mathbf{8 . 0}$ \\
\cline { 2 - 10 } & $Q^{\mathrm{I}}$ & 1.32 & 1.78 & 2.16 & 2.29 & 2.44 & 2.48 & 2.50 & 2.50 \\
\hline 2.5 & $Q^{\mathrm{II}}$ & 0.82 & 1.48 & 2.06 & 2.25 & 2.48 & 2.50 & 2.50 & 2.50 \\
\hline 2.5 & $Q^{\mathrm{I}}$ & 0.26 & 0.39 & 0.55 & 0.63 & 0.87 & 0.98 & 1.00 & 1.00 \\
\hline 1.0 & $Q^{\mathrm{II}}$ & 0.08 & 0.18 & 0.36 & 0.47 & 0.82 & 0.98 & 1.00 & 1.00 \\
\hline 1.0 & & & & & & & &
\end{tabular}

Point $M$ on curve 1 in Fig. 1 corresponds to the optimum value of anticorrosion expenditures. According to Eq. (12), EAC is a function of $k$. The relationship

$$
\mathrm{d}\left(E A C^{*}\right) / \mathrm{d} k=\left[1-\ln \left(K E A C^{*}\right)\right] /\left(E A C^{*}\right)^{2}
$$

allows one to estimate the most unfavourable anti-corrosion expenditures $\left(E A C^{* *}\right)$ : $E A C^{* *}=e / K$. Let us designate the corresponding coefficient of protective efficiency as $k_{\max }$. It equals 1.09 and 1.24 at $K=2.5$ and 2.2, respectively (points $P$ and $L$ in Fig. 2).

In the region where $E A C^{*}<E A C^{* *}$, which corresponds to $k<k_{\max }$ (low-efficiency anticorrosion protection), the optimum anticorrosion expenditures quickly decrease with decreasing $k$. If the latter inequality acquires an opposite sign (transition to highly-efficient anticorrosion protection methods), the decrease in $E A C^{*}$ with increasing protective efficiency $(E)$ of the method becomes comparatively small.

The value of $k$ can be obtained by solving the reverse task if the value of $E$ is known at a known $E A C$. As a rule, the latter is always the case. Statement of such a problem is entirely valid. 


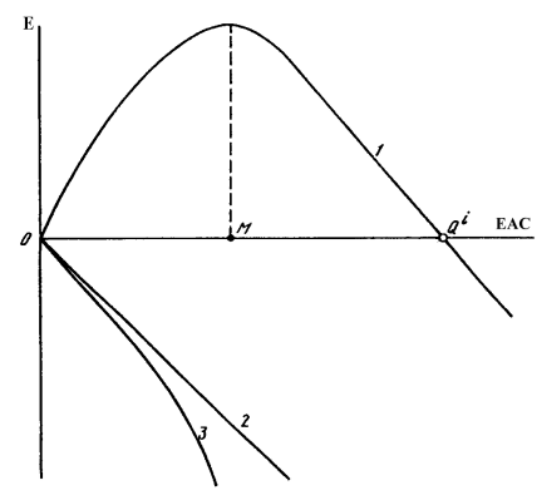

Fig. 1. Plot of economic efficiency $(E)$ of anticorrosion protection versus anticorrosion expenditures $(E A C) .1, k>0 ; 2, k=0 ; 3, k<0$.

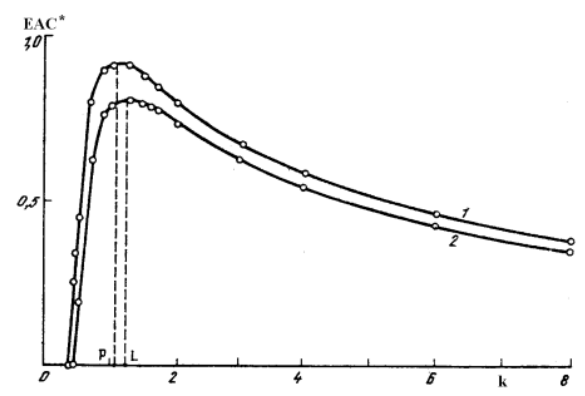

Fig. 2. Plot of the optimum $E A C^{*}$ value versus the anticorrosion protection efficiency. $1, K=$ $2.5 ; 2, K=2.2$.

Using Eq. (9), one can solve a number of specific problems, for instance:

1. Estimate the values of expenditures of two different anticorrosion protection methods where the $E$ value is the same. To do so, one can easily obtain the following equation:

$$
\exp \left(-k^{\mathrm{I}} E A C^{\mathrm{I}}\right)-\exp \left(-k^{\mathrm{II}} E A C^{\mathrm{II}}\right)+\left(E A C^{\mathrm{I}}-E A C^{\mathrm{II}}\right) / K=0
$$

The upper indices correspond to protection methods I and II. If $k^{\mathrm{I}}, k^{\mathrm{II}}$ and $E A C^{\mathrm{I}}$ are known, one can find the value of $E A C^{\mathrm{II}}$ by numeric calculation from Eq. (13) with desired precision without any difficulties.

2. Calculate the $k$ value for a new protection method that allows an $m$-fold decrease in $E A C$. At $E=$ const, the following equation can be obtained:

$$
k^{\mathrm{II}}=\left[\left(E A C^{\mathrm{II}} / K\right)(m-1)+\exp \left(-k^{\mathrm{I}} E A C^{\mathrm{II}} m\right)\right] / E A C^{\mathrm{II}}
$$


3. Calculate the value of anticorrosion expenditures allowing an $\mathrm{m}$-fold increase in $E$ for the same anticorrosion protection method. Let us designate $E A C^{\mathrm{II}} / E A C^{\mathrm{I}}=x$. Then the equation for the calculation is as follows:

$$
\left[1-\exp \left(-k^{\mathrm{I}} E A C^{\mathrm{I}}\right)-E A C^{\mathrm{I}} / K\right] /\left[1-\exp \left(-k^{\mathrm{I}} x\right)-x / K\right]-m=0
$$

It can be easily solved by numerical calculation in dialogue mode. For example, to increase $E 1.5$-fold at $k^{\mathrm{I}}=3, E A C^{\mathrm{I}}=0.2$ and $K=2.5, x=2.2($ here $m=1 / 1.5$ ).

The results of $E$ calculation as a function of $E A C$ are shown in Fig. 3. Curves 5 and 6 are in the negative $E$ zone. However, $E$ is negative with respect to $K_{\mathrm{dcl}}^{0}$ but positive with respect to $\left(K_{\mathrm{dcl}}^{0}+K_{\mathrm{icl}}^{0}\right)$, i.e., the overall protection is economically justified. Figure 4 shows how the form of suggested $K_{\text {scl }}=f(E A C)$ relationships affects $E-E A C$ plots.

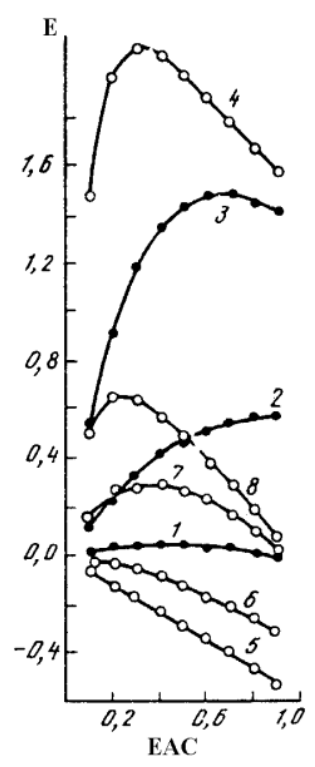

Fig. 3. Plot of economic efficiency $(E)$ of anticorrosion protection versus EAC. $1-4$, with the $\left(K_{\mathrm{dcl}}+K_{\text {icl }}\right)$ sum taken into account $(K=2.5) ; 5-8$, with direct corrosion losses $\left(K_{\mathrm{dcl}}\right)$ taken into account, $K=1 ; 1,5: k=0.5 ; 2,6: k=1.0 ; 3,7: k=3.0 ; 4,8: k=10.0$.

\subsection{Application of the power function for the relationship between $E, k$ and $E A C$}

Based on Eq. (8a), the dependence of $E$ on $K, k$ and $E A C$ has the form:

$$
E=K[1-(1-E A C) k-E A C / K]
$$

From Eq. (16):

$$
\begin{gathered}
\mathrm{d} E / \mathrm{d}(E A C)=K k(1-E A C)^{k-1}-1 \\
\mathrm{~d}^{2} E / \mathrm{d}(E A C)^{2}=-K k(k-1)(1-E A C)^{k-2}
\end{gathered}
$$




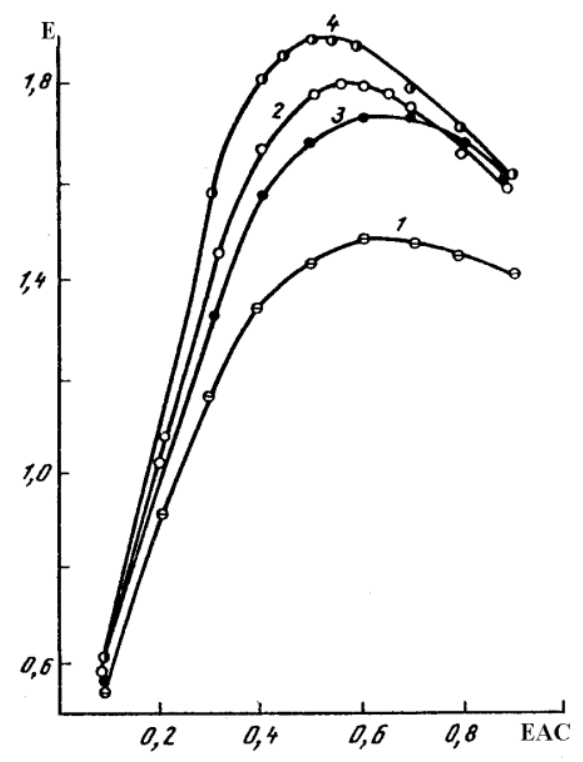

Fig. 4. Plot of economic efficiency $(E)$ of anticorrosion protection versus $E A C$ at $K=2.5$ and $k=3$. Equations used for the calculation of $K_{\text {scl }}=f(E A C): 1$, Eq. (7a); 2, Eq. (8a); 3, Eq. (7b); 4, Eq. (8b).

At $k>1$ and $E A C \leq 1, \mathrm{~d}^{2} E / \mathrm{d}(E A C)^{2}<0$. Therefore if an extremum is observed in the indicated range of $k$ and $E A C$ values, then it is definitely a maximum. In fact, function (16) has a maximum.

On the contrary, when $k<1$ (low-efficiency anticorrosion protection) and $E A C<1$, a minimum is observed. By equating $\mathrm{d} E / \mathrm{d}(E A C)$ to zero, one can obtain the dependence of $E A C^{*}$ on $K$ and $k$ :

$$
K k\left(1-E A C^{*}\right)^{k-1}-1=0
$$

The relationship between $E A C^{*}$ and $k$ is shown in Table 2.

Table 2. The relationship between $E A C$ and $k$ at $K=3$.

\begin{tabular}{cccccccccccc}
\hline $\boldsymbol{k}$ & $\mathbf{0 . 3}$ & $\mathbf{0 . 5}$ & $\mathbf{0 . 7}$ & $\mathbf{0 . 9}$ & $\mathbf{1 . 1}$ & $\mathbf{2 . 0}$ & $\mathbf{3 . 0}$ & $\mathbf{4 . 0}$ & $\mathbf{6 . 0}$ & $\mathbf{8 . 0}$ & $\mathbf{1 0 . 0}$ \\
\hline$E A C^{*}$ & 0.14 & -1.25 & -1.09 & -2.06 & 1.0 & 0.83 & 0.67 & 0.56 & 0.44 & 0.36 & 0.31 \\
\hline
\end{tabular}

The power function is less convenient for estimation of the economic efficiency of anticorrosion protection because unambiguous calculations can only be performed in the $k \geq 1$ range only, i.e., where $E$ is sufficiently high. If $k$ is small, this function gives $E A C^{*}$ values that lack physical meaning.

For a more accurate analysis, one ought to take the dependence of $k$ on $E A C$ into account. In the simplest case, it is convenient to use the expression $(1+E A C)^{k}$ instead of $k$, which leads to the equations: 


$$
\begin{gathered}
K_{\mathrm{scl}}=K(1-E A C)^{(1+E A C) k} \\
E=K\left[1-(1-E A C)^{(1+E A C) k}-E A C / K\right]
\end{gathered}
$$

If $E A C \leq 1$, function (18) has a maximum (Fig. 4). The value of $E A C^{*}$ can be obtained from the equation:

$$
K^{2} k\left(1-E A C^{*}\right)(1+E A C)^{k-1}\left[-\left(1+E A C^{*}\right)+\left(1-E A C^{*}\right) \ln \left(1-E A C^{*}\right)+1\right]=0,
$$

where the left-hand part characterizes $\mathrm{d} E / \mathrm{d}(E A C)$ multiplied by $(-1)$. The $k$ value can also be calculated from Eq. (16) and Eq. (18) by solving the inverse problem. For this purpose, it is easy to obtain relationships (19) and (20):

$$
\begin{gathered}
k=\ln (K-E-E A C) /[K(1-E A C)] \\
k=\ln (K-E-E A C) /\{(1+E A C) \ln [K(1-E A C)]\}
\end{gathered}
$$

\subsection{Taking the changes in corrosion losses into consideration}

If the corrosion losses decrease $m$-fold $(E A C \neq 0)$, one can write:

$$
m=K_{\mathrm{scl}}^{0} / K_{\mathrm{tcl}}
$$

Combining Eq. (2) and Eq. (5a), one can obtain:

$$
m=K_{\mathrm{scl}}^{0} /[K f(E A C)+E A C]
$$

Taking Eq. (7a) into account, we can write the base equation (as before, $K_{\text {dcl }}^{0}=1$ ):

$$
m=K[K \exp (-k E A C)+E A C]
$$

Use of Eq. (8) instead of Eq. (7a) or more accurate relationships

$$
\begin{gathered}
K_{\mathrm{scl}}=K \exp [-k E A C \exp (E A C)] \\
K_{\mathrm{scl}}=K(1-E A C)^{(1+E A C) k}
\end{gathered}
$$

leads to the equations:

$$
\begin{gathered}
m=K /\left[K(1-E A C)^{k}+E A C\right] \\
m=K /\{\operatorname{Kexp}[-k E A C \exp (E A C)]+E A C\} \\
m=K /\left[K(1-E A C)^{(1+E A C k}+E A C\right]
\end{gathered}
$$

As follows from Eq. (21): if $E A C=0$, then $m=1$; at $k=0$ and $E A C \neq 0, m<1$, i.e. in this case the corrosion losses increase with increasing $E A C$. From equality to zero of the derivative $\mathrm{d} m / \mathrm{d}(E A C)$ (Eq. (21)) one can obtain the dependence of the extremum point on $K$ and $k$ that has a form of Eq. (12).

By differentiating Eq. (22) with respect to $E A C$, one can obtain:

$$
\mathrm{d} m / \mathrm{d}(E A C)=-K\left[1-k K(1-E A C)^{k-1} /\left[K(1-E A C)^{k}+E A C\right]^{2},\right.
$$

whence it follows at $\mathrm{d} m / \mathrm{d}(E A C)=0$ : 


$$
E A C^{*}=1-(k K) 1 /(1-k)
$$

\subsection{Taking inflation and deflation into consideration}

Inflation has to be taken into account if there is a long time interval between the anticorrosion expenditures and the observed economic effect. The value of $K$ remains the same in this case.

For instance, let $E$ be estimated $\tau$ time units (months, years) later after the investment of the $E A C$. Let us assume that the average inflation (deflation) coefficient during the interval $\tau$ equals $\alpha$. It is measured by portions of the expenditures and can be both larger and smaller than zero. Then $K_{\mathrm{scl}}^{0}, K_{\mathrm{dcl}}^{0}$ and $K_{\mathrm{icl}}^{0}$ change by $\alpha \tau K_{\mathrm{scl}}^{0}, \alpha \tau K_{\mathrm{dcl}}^{0}$, and $\alpha \tau K_{\mathrm{icl}}^{0}$, respectively, over the period considered. Taking these values into account, Eq. (9) turns to:

$$
E=(1+\alpha \tau) K\{1-\exp (-k E A C)-E A C /[K(1+\alpha \tau)]\},
$$

whence it follows:

$$
\begin{aligned}
& \mathrm{d} E / \mathrm{d}(E A C)=(1+\alpha \tau) K k \exp (-k E A C)-1 \\
& \mathrm{~d}^{2} E / \mathrm{d}(E A C)^{2}=-(1+\alpha \tau) K k^{2} \exp (-k E A C)
\end{aligned}
$$

The second derivative $\mathrm{d}^{2} E / \mathrm{d}(E A C)^{2}<0$ if $(1+\alpha \tau)>0$, i.e. for all inflation $(\alpha>0)$ and deflation processes $(\alpha<0)$ as long as $\alpha \tau<1$. Therefore, the extremum is a maximum under these conditions and the optimum $E A C$ value equals:

$$
E A C^{*}=f(K, k, \alpha, \tau)
$$

From the condition $\mathrm{d} E / \mathrm{d}(E A C)=0$ one can obtain:

$$
E A C^{*}=\frac{\ln [(k K(1+\alpha \tau)]}{k}
$$

It is also desirable to take estimates of $\alpha$ within shorter time intervals into account. Then Eq. (25) takes the form:

$$
E=\left(1+\alpha_{1} \tau_{1}+\ldots .+\alpha_{i} \tau_{i}\right) K\left\{1-\exp (-k E A C)-E A C /\left[K\left(1+\alpha_{1} \tau_{1}+\ldots .+\alpha_{i} \tau_{i}\right)\right]\right\},
$$

where $\tau=\tau_{1}+\ldots .+\tau_{i}$.

To obtain more precise relationships, compound interest has to be used. Then Eq. (26) turns into Eq. (27):

$$
E=(1+\alpha)^{\tau} \mathrm{K}\left\{1-\exp (-k E A C)-E A C /\left[K(1+\alpha)^{\tau}\right]\right\},
$$

whence it follows:

$$
\begin{aligned}
& \mathrm{d} E / \mathrm{d}(E A C)=(1+\alpha)^{\tau} K k \exp (-k E A C)-1 \\
& \mathrm{~d}^{2} E / \mathrm{d}(E A C)^{2}=-(1+\alpha)^{\tau} K k^{2} \exp (-k E A C)
\end{aligned}
$$

In this case $\mathrm{d}^{2} E / \mathrm{d}(E A C)^{2}<0$ as long as $\alpha>-1$ under deflation conditions.

$$
E A C^{*}=\frac{\ln \left[\left(k K(1+\alpha)^{\tau}\right]\right.}{k}
$$


If $\alpha \ll 1$, one can obtain after expanding the function $(1 \pm \alpha)^{\tau}$ and $\mathrm{e}^{\alpha \tau}$ in series:

$$
(1 \pm \alpha)^{\tau}=1 \pm \alpha \tau, \mathrm{e}^{\alpha \tau}=1 \pm \alpha \tau, \text { then }(1 \pm \alpha)^{\tau}=\mathrm{e}^{\alpha \tau} .
$$

Then Eq. (27) takes the form:

$$
E=\exp (\alpha \tau) K\{1-\exp (-k E A C)-E A C /[K \exp (\alpha \tau)]\}
$$

and

$$
E A C^{*}=\frac{\ln [k K \exp (\alpha \tau)]}{k}
$$

\section{Conclusions}

The suggested method for the estimation of the economic efficiency of anticorrosion protection is solely based on the quantities available to a consumer performing a systematic calculation of revenues and anticorrosion expenditures. This method allows one to estimate the efficiency of the anticorrosion protection methods already used or suggested, including the use of corrosion inhibitors, for industrial application.

\section{References}

1. N. A. Stepanov, N. Ya. Savelyev and O. L. Figovsky, Anticorrosion service at enterprises, Moscow, Metallurgiya, 1987 (in Russian).

2. A method to determine the economic efficiency of the use of the corrosion inhibitors in the gas industry, Moscow, Publishing house of VNIIGas, 1981, 39 (in Russian).

3. G. S. Keselman, Economic efficiency of the corrosion prevention in the oil industry Moscow, Nedra publishing house, 1988 (in Russian).

4. G. S. Keselman, Ya. M. Kolotyrkin and V. M. Novakovskyi, Some economic aspects of the corrosion problem and anticorrosion protection), Moscow, Publishing house of VNIIGas, 1979 (in Russian).

5. N. V. Shell, A. P. Pozdnyakov and V. I. Vigdorovich, Vestnik Tambovskogo Universiteta, Estestvennye i tekhnicheskie nauki, 1999, 4, no. 1, 59 (in Russian).

6. V. I. Vigdorovich and N. V. Shell, Zashch. Met., 1993, 29, 953 (in Russian).

7. V. I. Vigdorovich and N. V. Shell, Vestnik Tambovskogo Universiteta, Estestvennye $i$ tekhnicheskie nauki, 1997, 2, no. 2, 182 (in Russian).

8. V. I. Vigdorovich and N. V. Shell, Materials of the International Conference "Mathematical Methods in Economics", Vestnik Tambovskogo Universiteta, Estestvennye i tekhnicheskie nauki, 2000, 5, no. 4, 512 (in Russian).

9. P. Elliot, C. M. Fewler, B. Johnson and C. C. Wood, Agricultural Engineer, 1983, no. $1,13$.

10. A. E. Severnyi, Sci. Doctor Diss., Moscow, 1988 (in Russian). 